\title{
ICE Testing Process Decomposition
}

\author{
${ }^{1}$ Lenar A. Galiullin, ${ }^{2}$ Shafik Sh. Khuzyatov \\ 1-2 Kazan Federal University \\ Email: galilenar@yandex.ru
}

Received: 23 ${ }^{\text {rd }}$ July 2019, Accepted: 10 ${ }^{\text {th }}$ August 2019, Published: $31^{\text {st }}$ August 2019

\begin{abstract}
The solution of the problem of designing, developing and further using automated test systems (ATS) of internal combustion engines (ICE) involves, first of all, analyzing a number of important requirements for the development of technical, mathematical, software, information, linguistic and organizational-methodological support of an automated system.

Currently, the need for widespread implementation and operation of automated systems in real test conditions of stations of production plants and design and research institutes imposes some restrictions on the design of automated tuning tools for real technologies of testing various types and modifications of internal combustion engines. This circumstance is based on a sufficiently large number of tested engines, units and assemblies of various modifications, as well as the need to gradually eliminate errors in existing algorithms, including the creation of new test algorithms, in particular, when conducting research and development tests of engines.

Requirements of real engine operating conditions cause a constant increase in the technical level of the modifications produced and lead to the fact that the nature of the cost of testing when designing new types of engines increases each time. These costs are the highest in the absence of a relationship between the levels of production automation and research and development. The result is that the automation of technological processes for testing internal combustion engines is one of the most important tasks of increasing the technical level of production and the quality of the engines produced.
\end{abstract}

\section{Keywords}

Decomposition; Engine; Test; Programming; Controller; Model.

\section{Introduction}

The growth of the power supply of all industries throughout the world has caused a wide development of enginebuilding, including the production of engines of various types, and therefore the problem of improving them is of great importance [1].

The solution to the problem of improvement is more dependent on the compliance of the fuel injection process with the work process that takes place in the engines [2]. For continuous management and control of the process of the internal combustion engine, it is necessary to implement a controller [3]. The electronic control unit (ECU) allows you to control solenoids injectors. It monitors and processes the various input signals of the measuring sensors [4]. The basic data are the engine's crankshaft speed and load, which the driver can directly influence with the accelerator pedal [5]. Controlled parameters also include air temperature, fuel, coolant and pressure of the turbocharger [6]. These basic functions can be complemented by a variety of other operations designed to enhance convenience [7]. The controller must meet the stringent requirements for the reliability of work compensation and the identification of malfunctions in the operation of individual components. It is also intended to facilitate the diagnostics of malfunctions of the engine and its fuel injection system [8].

To check the quality of the assembly and test ICEs equipped with an electronic controller, it is necessary to introduce a technical complex at the manufacturing plant that provides automatic control of the ICE technological modes of operation [9].

These systems provide the following functions:

- $\quad$ work at the pace of technological processes (in real time);

- implementation of automatic control and management of the process of testing the engine;

- execution of technical control protocols and reports on the implementation of the production program;

- implementation of the specified cyclogram of technical control for each type of engine;

- warning of abnormal and emergency situations when testing ICE.

Functions performed by ICE test systems:

- verification of operability of sensors of primary parameters;

- performance testing of actuators;

- checking the correctness of control algorithms;

- checking the availability and accuracy of informational messages;

- registration of failures of software and hardware;

- recognition of failures that are not automatically detected;

- $\quad$ analysis of the $\log$ of failures of software and hardware; 
- control of the correct functioning of software and hardware.

\section{Methods}

The rapid progress of software and its rapid penetration into almost all areas of human activity are particularly noticeable in the example of the automotive industry [10]. Currently, auto-electronics is undergoing evolutionary development to various problems, which gives grounds for assuming the birth of revolutionary engineering developments in the near future [11].

Integrated circuits on semiconductor elements have revolutionized the automotive industry, especially in the management of automotive units and the car as a whole [12]. Nowhere in the world is not produced a single car without electronic devices [13]. The main ones are voltage regulators, transmission control devices, fuel injection, brake system, steering, suspension [14].

The first micro-computers began to be used to control the ignition timing (1976 in the Misar systems of General Motors) [15]. Due to the high control accuracy, it became possible to significantly improve engine performance [16].

In 1980, electronic dashboards, suspension control systems, automatic air conditioners, radio receivers with electronic tuning, multifunctional information systems with displays on cathode-ray tubes, etc., appeared [17]. The same ECUs diagnose themselves [18].

All electronic components by function can be classified into three main control systems: the engine; transmission and chassis; cabin equipment [19].

Currently in the world developed and commercially produced a wide variety of engine management systems [20]. These systems, according to the principle of action, have much in common, but they differ significantly. By appointment, they are monofunctional and complex. In complex systems, one electronic unit controls several subsystems: fuel injection, ignition, valve timing, self-diagnostics, etc. [21]. In monofunctional systems, the ECU sends signals only to the injection system [22]. The distribution of fuel distinguishes between multi-point and central injection. With multipoint injection, one nozzle is installed for each cylinder, and with a central injection there is one nozzle for all cylinders [23].

In addition, the difference lies in the method of injection. Injection can be carried out continuously and pulses. With a constant supply of fuel, its quantity changes due to a change in pressure in the fuel line, and during a pulse flow, due to the duration of the pulse and its frequency. Thus, in one injection, a full portion of the fuel or part of it (usually half) can be supplied. If for each revolution of the crankshaft one fuel is injected into each cylinder, this injection is called synchronous.

A comprehensive engine management system ensures optimum performance by controlling fuel injection, ignition timing, engine speed at idle and diagnostics.

Updating the equipment of modern cars can be shown in the form of many small, quickly following each other steps, and often this does not threaten auto manufacturers with additional financial costs for electronics [24]. Currently, electronics are increasingly being used, including for controlling the combustion of fuel in internal combustion engines, in monitoring devices for the state of individual systems and devices, for controlling actuators and the like.

Currently, new developed car models have additionally begun to install completely non-standard full-time automated systems, these include an automatic assistance system with software, including: satellite navigation system; sound and radar systems to protect the car from theft and collisions; systems to improve the comfort and safety of people in the cabin; cruise control system, etc.

At the same time, searches are carried out for the most optimal automatic message processing technology in onboard automated systems. The sometimes called "linguistic functional transducers" have been developed and found to work with fuzzy subsets of linguistic variables expressed by separate words or whole sentences in natural (English) or artificial (computer) language. With some complication of logical and arithmetic operations in a micro-computer, this provides an increase in the accuracy and adequacy (speed) of processing system messages. Therefore, the interface has become much more complicated and it has become necessary to maintain the CAN protocol in a multiplex system.

The use of an electronic engine management system has the following advantages:

- ensures optimal fuel supply to the engine at all operating conditions, resulting in reduced fuel consumption, while maintaining the dynamic characteristics of the car;

- due to more complete combustion of the fuel, as well as the use of a catalyst, the emission of harmful substances along with exhaust gases is reduced;

- as the engine electronic control system detects and writes to the fault memory, search and fault detection is greatly accelerated;

- the engine control system at all operating conditions sets the optimum ignition timing, time and amount of fuel supplied to the engine. Also, the engine management system interacts with other vehicle systems, in particular, with an automatic transmission control system or with an anti-theft system.

The components of the engine management system are reliable and practically maintenance free. During maintenance, it is only necessary to replace the air filter and spark plugs. Since it is necessary to use complex and expensive equipment to check, adjust and repair the engine management system, this work must be done at a specialized service station. Today there are many standards for communication protocols between the computer and the test system. The list of ISO and SAE international standards for diagnostics:

- ISO 9141: Requirements for sharing digital information

- $\quad$ ISO 9141-2: Requirements for sharing digital information 
- ISO 9141-3: Setting up the connection between the vehicle and OBD II

- ISO 14230-1: Protocol 2000 - Physical Layer

- ISO 14230-2: Protocol 2000 - Communication Channel

- ISO 14230-3: Protocol 2000 - Application Layer

- ISO 14230-4: Protocol 2000 - Requirements for Emission Systems

- SAE J1850: Class B. Data Network Interface

- SAE J2012: Recommended Practice for Diagnostic Trouble Code Definitions

- SAE J1930: Electrical / electronic diagnostic system: detection speed, abbreviations and acronyms

- SAE J2178: Class B. Data Network Communications

- SAE J1978: OBD II Scanning Tools

- SAE J1979: Diagnostic Test Methods

- SAE J2201: Universal interface for OBD II viewing tools.

\section{Results and Discussion}

The creation of an automated system is carried out on the basis of an object-oriented approach. The advantage of this approach is the interface for loading the test project and maintaining the OBD-II data exchange protocol between the electronic control unit.

To create the structure of the project model, it is necessary to determine the initial structure of the testing process, which displays the scheme of operation of the automated test system, and also to establish the functional features of the specified modes.

During testing, all information is stored in the memory of the ECU in the form of a table, the structure of which is presented in Table 1.

\begin{tabular}{|c|c|c|c|c|c|}
\hline № & Parameter 1 & Parameter 2 & Parameter 3 & $\ldots$ & Parameter $\mathrm{m}$ \\
\hline 1 & A11 & A21 & A31 & $\ldots$ & Am1 \\
\hline 2 & A12 & A22 & A32 & $\ldots$ & Am2 \\
\hline 3 & A13 & A23 & A33 & $\ldots$ & Am3 \\
\hline & $\ldots$ & $\ldots$ & $\ldots$ & $\ldots$ & $\ldots$ \\
\hline S & A1S & A2S & A3S & $\ldots$ & AmS \\
\hline
\end{tabular}

Table 1: Summary Table of Parameters

where: $A i j$ - value of engine parameter;

$M$ - the number of measured parameters;

$S$ - the number of test points measured during the test.

For a more accurate selection of test modes, it is necessary to take into account the main characteristics of the internal combustion engine that require special monitoring. When testing ICE, the following parameters should be checked:

- current fuel delivery options;

- regulation of idling;

- functionality of the air inflation sensor;

- transition of the fuel mixture from the poor to the saturated mode.

Initially, the engine and vehicle parameters file is loaded into the electronic control unit. After loading, the internal combustion engine is launched, with subsequent impact on the performing devices for testing the internal combustion engine. During the test, information is collected from the sensors. The obtained information is displayed on the monitor.

\section{Summary}

As a result of this work, an automated engine test system was developed.

In the course of the project, an analysis of the existing ICE test systems was carried out, which showed the need to develop an automated test system, given the small competition. To create the top-level software, the structure of the testing process was set based on the design and technological parameters of the internal combustion engine. In the process of developing the structure of the test system, the main groups of modes were defined. To implement the test modes, the constants of the variables assigned to the groups were determined in accordance with their functional purposes, which eliminates the presetting of the test system project tree. Based on the given structure of the testing process, the top-level software was implemented.

The automated test system is based on an object-oriented approach. The advantage of this program is a convenient interface for loading the test project, as well as support for the OBD-II data exchange protocol with the electronic control unit.

\section{Conclusions}

Based on the above, we can conclude that the developed test system allows us to effectively test an engine. Of particular interest is the possibility of comparing the compliance of the current parameters obtained as a result of tests with the parameters specified in the design of the engine and the vehicle as a whole. 


\section{Acknowledgements}

The work is performed according to the Russian Government Program of Competitive Growth of Kazan Federal University.

\section{References}

[1] M. Shah, V. Gaikwad, S. Lokhande, S. Borhade, "Fault identification for I.C. engines using artificial neural network," Proceedings of 2011 International Conference on Process Automation, Control and Computing, PACC 2011, art. no. 5978891, 2011.

[2] L. Guihang, W. Jian, W. Qiang, S. Jingui, "Application for diesel engine in fault diagnose based on fuzzy neural network and information fusion," 2011 IEEE 3rd International Conference on Communication Software and Networks, ICCSN 2011, art. no. 6014398, pp. 102 - 105, 2011.

[3] Y. Yu, J. Yang, "The development of fault diagnosis system for diesel engine based on fuzzy logic," Proceedings - 2011 8th International Conference on Fuzzy Systems and Knowledge Discovery, FSKD 2011, 1, art. no. 6019556, pp. 472 - 475, 2011.

[4] L.A. Galiullin, R.A. Valiev, "Modeling of internal combustion engines test conditions based on neural network," (2016) International Journal of Pharmacy and Technology, 8 (3), pp. 14902-14910.

[5] D. Wei, "Design of Web based expert system of electronic control engine fault diagnosis," BMEI 2011 Proceedings 2011 International Conference on Business Management and Electronic Information, 1, art. no. 5916978, pp. 482 - 485, 2011.

[6] L.A. Galiullin, R.A. Valiev, “An automated diagnostic system for ICE,” (2018) Journal of Advanced Research in Dynamical and Control Systems, 10 (10 Special Issue), pp. 1767-1772.

[7] R. Ahmed, M. El Sayed, S.A. Gadsden, J. Tjong, S. Habibi, "Automotive internal-combustion-engine fault detection and classification using artificial neural network techniques," IEEE Transactions on Vehicular Technology, 64 (1), № 6803044, pp. 21-33, 2015.

[8] L.A. Galiullin, R.A. Valiev, "Method for neuro-fuzzy inference system learning for ICE tests," (2018) Journal of Advanced Research in Dynamical and Control Systems, 10 (10 Special Issue), pp. 1773-1779.

[9] J. Chen, R. Randall, N. Feng, B. Peeters, H. Van Der Auweraer, "Automated diagnosis system for mechanical faults in IC engines," 10th International Conference on Condition Monitoring and Machinery Failure Prevention Technologies, CM 2013 and MFPT 2013, 2, pp. 892-903, 2013.

[10] L.A. Galiullin, R.A. Valiev, "Modeling of internal combustion engines by adaptive network-based fuzzy inference system," (2018) Journal of Advanced Research in Dynamical and Control Systems, 10 (10 Special Issue), pp. 1759-1766.

[11] J. Chen, R. Randall, B. Peeters, W. Desmet, H. Van Der Auweraer, "Neural network based diagnosis of mechanical faults in IC engines," Institution of Mechanical Engineers - 10th International Conference on Vibrations in Rotating Machinery, pp. 679-690., 2012.

[12] L.A. Galiullin, R.A. Valiev, "Optimization of the parameters of an internal combustion engine using a neural network," (2018) Journal of Advanced Research in Dynamical and Control Systems, 10 (10 Special Issue), pp. 1754-1758.

[13] J.-D. Wu, C.-K. Huang, Y.-W. Chang, Y.-J. Shiao, "Fault diagnosis for internal combustion engines using intake manifold pressure and artificial neural network," Expert Systems with Applications, 37 (2), pp. 949-958, 2010.

[14] Lenar A. Galiullin, Rustam A. Valiev, Lejsan B. Mingaleeva, "Development of a Neuro-Fuzzy Diagnostic System Mathematical Model for Internal Combustion Engines,” HELIX, Volume: 8, Issue: 1, Pages: 2535-2540, Published: 2018.

[15] Lenar A. Galiullin, Rustam A. Valiev, "Mathematical modelling of diesel engine testing and diagnostic regimes," Turkish Online Journal of Design Art and Communication, Volume: 7, Pages: 1864-1871, Published: DEC 2017.

[16] Lenar A. Galiullin, Rustam A. Valiev, "Diagnosis System of Internal Combustion Engine Development," Revista Publicando, Volume: 4, Issue: 13, Pages: PR128-PR137, Part: 2, Published: 2017.

[17] D. Danfeng, M. Yan, G. Xiurong, "Application of PNN to fault diagnosis of IC engine," 2009 2nd International Conference on Intelligent Computing Technology and Automation, ICICTA 2009, 2, № 5287738, pp. 495-498, 2009.

[18] L.A. Galiullin, R.A. Valiev, "Diagnostics Technological Process Modeling for Internal Combustion Engines," 2017 International Conference on Industrial Engineering, Applications and Manufacturing (ICIEAM) Published: 2017.

[19] L.A. Galiullin, R.A. Valiev, L.B. Mingaleeva, "Method of internal combustion engines testing on the basis of the graphic language,” Journal of Fundamental and Applied Sciences, Volume: 9, Special Issue: SI, Supplement: 1, Pages: 1524-1533, Published: 2017.

[20] L.A. Galiullin, "Development of Automated Test System for Diesel Engines Based on Fuzzy Logic,” IEEE 2016 2ND International Conference on Industrial Engineering, Applications And Manufacturing (ICIEAM) Published: 2016. 
[21] Lenar A. Galiullin; Rustam A. Valiev, "Control vector for ice automated test and diagnostic system," DILEMAS CONTEMPORANEOS-EDUCACION POLITICA Y VALORES, №6 SI, article №95, 2018.

[22] R.A. Valiev, A.Kh. Khairullin, V.G. Shibakov, "Automated Design Systems for Manufacturing Processes," Russian Engineering Research, 35(9). pp. 662 - 665, 2015.

[23] Lenar A. Galiullin; Rustam A. Valiev, Khairullin A. Haliullovich, "Method for modeling the parameters of the internal combustion engine,” IIOAB JOURNAL, №9, pp. 83-90, 2018.

[24] Lenar A. Galiullin; Rustam A. Valiev, "Internal combustion engine fault simulation method," IIOAB OURNAL, №9, pp. 91-96, 2018. 